\title{
Polarization characteristics of the Crab pulsar's giant radio pulses at HFCs phases
}

\author{
A. Słowikowska*, A. Jessner ${ }^{\dagger}$, B. Klein ${ }^{\dagger}$ and G. Kanbach** \\ *Nicolaus Copernicus Astronomical Center, Rabiańska 8, 87-100 Toruń, Poland \\ ${ }^{\dagger}$ Max Planck Institute for Radioastronomy, Auf dem Hügel 69, D-53121 Bonn, Germany \\ ** Max Planck Institute for Extraterrestrial Physics, Postfach 1312, D-85741 Garching, Germany
}

\begin{abstract}
We discuss our recent discovery of the giant radio emission from the Crab pulsar at its high frequency components (HFCs) phases and show the polarization characteristic of these pulses. This leads us to a suggestion that there is no difference in the emission mechanism of the main pulse (MP), interpulse (IP) and HFCs. We briefly review the size distributions of the Crab giant radio pulses (GRPs) and discuss general characteristics of the GRP phenomenon in the Crab and other pulsars.
\end{abstract}

\section{INTRODUCTION}

The occurrence of sporadic emission of intense pulses from the Crab pulsar (PSR B0531+21) has been known since its discovery in 1968 by Staelin and Reifenstein [1]. It should be noticed that the pulsar would not have been discovered if it had not
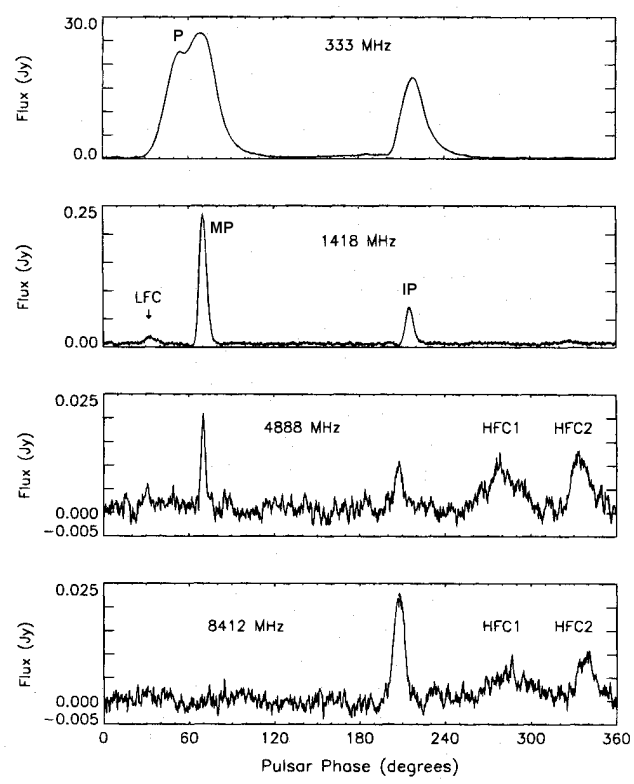

FIGURE 1. The aligned, average radio intensity profiles of the Crab pulsar obtained at $0.33,1.4,4.9$ and $8.4 \mathrm{GHz}$ with VLA showing several components: the main pulse (MP), interpulse (IP), precursor (P), low frequency component (LFC), and two broad high frequency components (HFC1, HFC2), waxing and waning with radio frequency. 


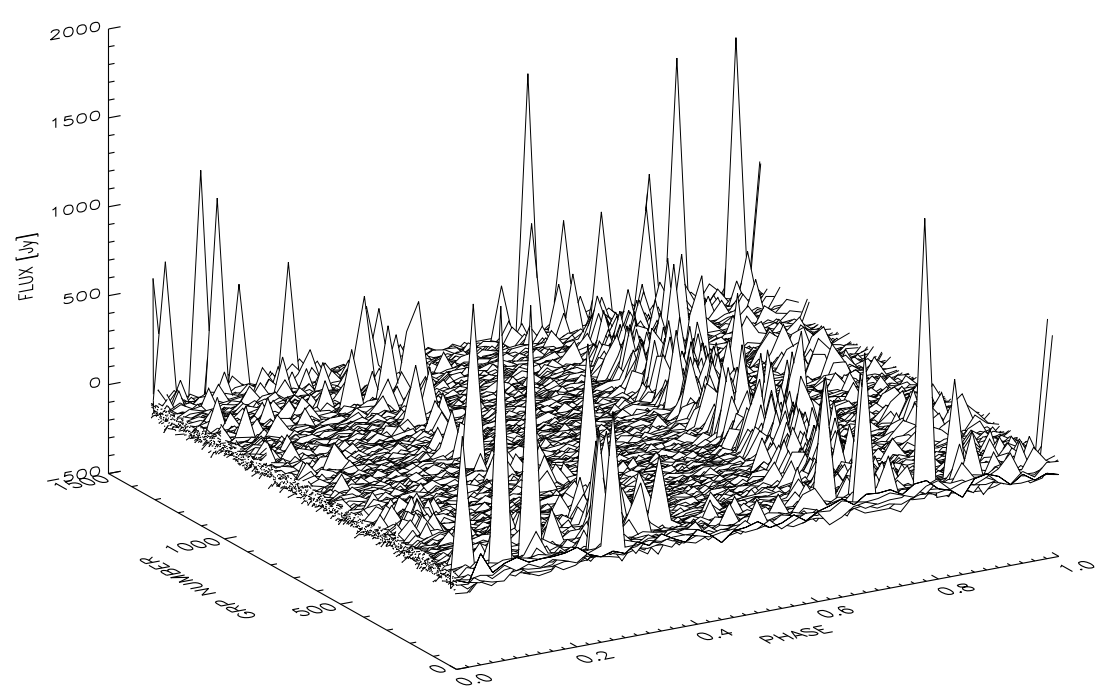

FIGURE 2. A train of strong giant pulses at $8.35 \mathrm{GHz}$, sum of the right and left handed circular polarisation signals. Results obtained during $6.7 \mathrm{hr}$ of observation with the $100 \mathrm{~m}$ Effelsberg telescope. Location of the Crab's pulsar radio components: HFC2: 0.05, precursor: 0.2, MP: 0.3, IP: 0.68, HFC1: 0.9 .

showed the giant radio pulses phenomenon. For over 25 years, only the Crab pulsar was known to emit giant radio pulses. Moreover, for over 35 years, it was thought that only the main pulse and interpulse exhibit the GRP phenomenon (e.g. [2] - discovery of GRPs at IP phase, [3], [4], [5]). These two components have counterpart non-thermal emission from the infrared to the gamma-ray energies. Until this year ([6]) the Crab's giant pulses were not seen in the radio precursor nor at the phases of the high radio frequency components (HFC1 and HFC2, see Fig. 1), that were first identified by Moffett and Hankins [7].

\section{OBSERVATIONS}

Observations with the Effelsberg 100-m radio telescope began on 25 November 2003 and ended on 28 November 2003. We used a secondary focus cooled HEMT receiver with a center frequency of $8.35 \mathrm{GHz}$, providing two circularly polarized IF signals with a system temperature of $25 \mathrm{~K}$ on both channels. With a sky temperature of $8 \mathrm{~K}$, and a contribution of $33 \mathrm{~K}$ from the Crab nebula, the effective system temperature was $70 \mathrm{~K}$. Two detection systems were used. First, a polarimeter with $1.1 \mathrm{GHz}$ bandwidth detected total power of the left-hand and right-hand circularly polarized signals (LHC and RHC respectively) as well as $\cos \angle($ LHC, RHC) and $\sin \angle($ LHC, RHC). These four signals were then recorded by the standard Effelsberg Pulsar Observation System (EPOS). With the Crab pulsar's dispersion measure of $56.8 \mathrm{pc} \mathrm{cm}^{-3}$, we had an un-dedispersed time resolution of $t_{\text {sample }}=890 \mu \mathrm{s}$, while our sampling resolution was fixed at $\sim 640 \mu \mathrm{s}$. 
EPOS was therefore set to continuously record data blocks containing 20 periods divided into 1020 phase bins for all four signals. The minimum detectable flux per bin was $\Delta S_{\text {min }}=0.117 \mathrm{Jy}$. Considering the dispersion pulse broadening, the detection limit for a single GRP of duration $\tau_{g r p}=3 \mu$ s would be $\Delta S_{\text {min }} t_{\text {sample }} / \tau_{g r p}=25 \mathrm{Jy}$. Selected GRPs are shown in the Fig. 2,

About $2.4 \times 10^{6}$ periods were observed with EPOS. The results presented here are based on a one-third of all observed rotations only. This restriction was due to insufficient $\mathrm{S} / \mathrm{N}$ in the remaining data. Because of the Crab pulsar's weak signal at $8.35 \mathrm{GHz}$ and the strong nebula background, ordinary single pulses were not observable with Effelsberg at that frequency. It takes about 20 min integration to assemble a mean profile at that frequency. The giant pulses come in short episodes, about 5 to 20 minutes in duration, and appear extremely prominent during such burst phases.

In our subsequent analysis, we set a threshold level of $5 \mathrm{rms}=125 \mathrm{Jy}$ on the sum of RHC and LHC for the same phase bin to count as a detection of a giant pulse. More than 1300 giant pulses were detected that way (Fig. 2) and their arrival phases were computed by using the TEMPO $^{1}$ pulsar timing package. The data were aligned using the current Jodrell Bank timing model. They were found to be a perfect match to the time of arrivals (TOAs) obtained at Jodrell Bank before and after the Effelsberg observing session. We found that the giant pulses occur at all those phases where the radio components of the Crab pulsar emission are observed.

\section{POLARIZATION}

The average polarization characteristics of 900 GRPs in some aspects are similar to already published high radio frequencies observations, but in some aspects we do observe significant differences. For all authors (this work, [8] and [9]) relative offset of the position angle (P.A.) between IP and HFCs is on the level of 40-45 degree. However, the P.A. for the IP, and at the same time for the HFCs differ for all authors. They are as follow, for the IP: $30^{\circ}, 0^{\circ},-30^{\circ}$, and for the HFCs: $60^{\circ}$ to $70^{\circ}, 45^{\circ}, 0^{\circ}$ according to [8], [9] and this work, respectively. Moreover, there is some discrepancy in the degree of polarization. Almost 100\% linear polarization of all three components has been derived by Karastergiou et al. [9], whereas in [8] the IP is polarized in only 50\% and HFCs in $80-90 \%$. In our work all components are polarized at the same level of $70-80 \%$. This may be due to the time varying contribution of the nebula [10] to the rotation measure of the pulsar ([10]: RM=-43 $\mathrm{rad} \mathrm{m}^{-2}$, [8]: $\mathrm{RM}=-46.9 \mathrm{rad} \mathrm{m}^{-2},[11]: \mathrm{RM}=-58 \mathrm{rad} \mathrm{m}^{-2}$ ). No abrupt sweeps in P.A. are found within pulse components.

The study of radio polarization has not improved essentially our knowledge of the emission and magnetic field geometry: e.g., by fitting the rotation vector model to the radio polarization data uttery opposite conclusions had been reached ([8], [9]). Moffett and Hankins [8] found that the angle between the rotation and magnetic axes is $\alpha=56^{\circ}$, while Karastergiou et al. [9] got nearly aligned rotator with $\alpha=4^{\circ} \pm 1^{\circ}$. They obtained

1 http://pulsar.princeton.edu/tempo 

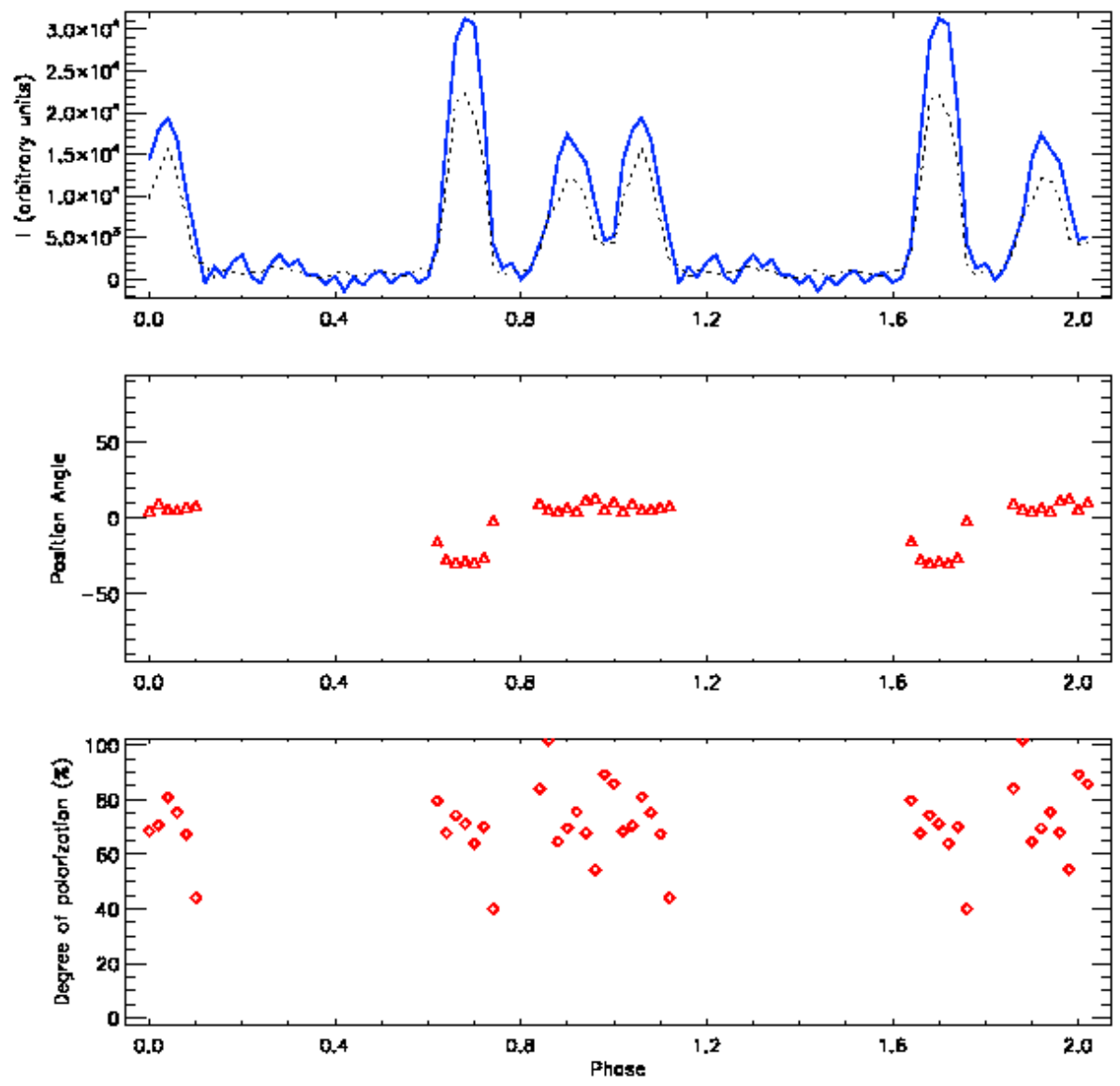

FIGURE 3. The average intensity profile (top), the position angle (after Faraday correction, middle), and the degree of polarization (bottom) of the 874 Crab's GRPs recorded at $8.35 \mathrm{GHz}$. Location of the Crab's pulsar radio components: HFC2: 0.05, precursor: 0.2, MP: 0.3, IP: 0.68, HFC1: 0.9.

different results for the impact angle as well.

In the Fig. 4 the position angle of selected 874 GRPs as a function of rotational phase of the Crab pulsar is shown. In these polarization characteristics of single pulses we do not observe any sudden changes of the P.A. within the separated components, as it has been noticed for PSR B0329+54 by Edwards and Stappers [12].

\section{GRPS DISTRIBUTION}

For all giant pulses, i.e. regardless of their phases, the histogram of their peak strengths at $8.35 \mathrm{GHz}$ can be described by a power-law with a slope $\sim-3.34 \pm 0.19$ ([6]). This result is consistent with the results obtained by others (e.g. [3]). Proportional contribution of a number of GRPs to four different phase components is as follow: IP - 80\%, MP $9 \%$, HFC1 - 7\%, and HFC2 - 4\%. Because of the limited statistics we could make a model $(S / N)^{\alpha}$ fit only for the IP component, where a power-law index $\sim-3.13 \pm 0.22$ 


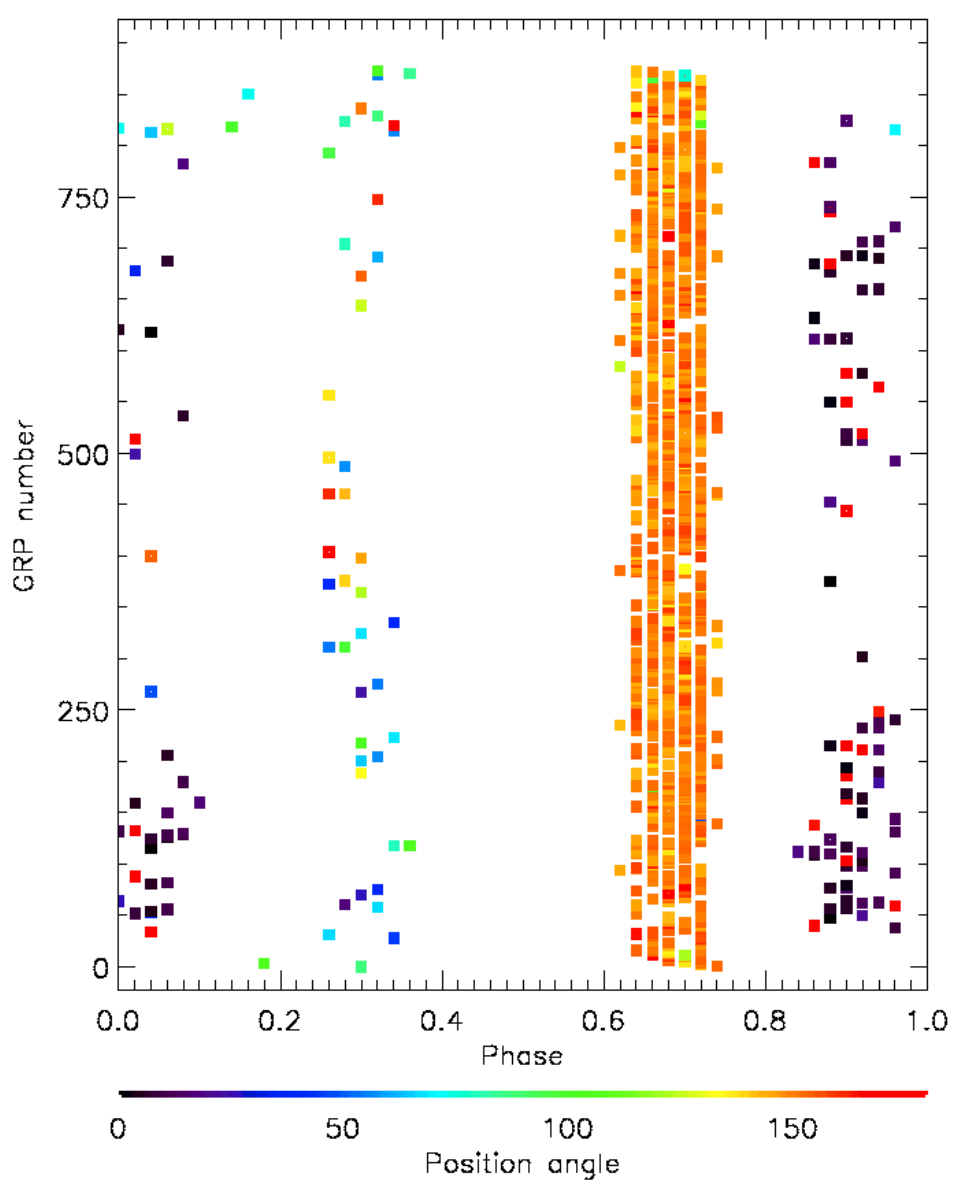

FIGURE 4. The position angle of selected 874 GRPs as a function of rotational phase of the Crab pulsar. P.A. is plotted only for points above $4.5 \sigma$ of the off-pulse noise. Location of the Crab's pulsar radio components: HFC2: 0.05, precursor: 0.2, MP: 0.3, IP: 0.68, HFC1: 0.9. (Full colour version can be found at the astro-ph service).

was found, and it is consistent with the value of $\sim-2.9$ presented by Cordes et al. [5]. The MPs from the Crab pulsar at $146 \mathrm{MHz}$ are distributed according to a power-law with the exponent of -2.5 , whereas the IPs with -2.8 [13]. For comparison at $800 \mathrm{MHz}$ the distribution (regardless of the phases of GRPs) has a slope of -3.46 [3]. It should be noticed that at low radio frequency the main contribution to the number of GRPs comes from main pulses. So far, there is no evidence that the distributions of numbers of GRPs for the HFC1 and HFC2 differ from each other. However, their slopes seems to be steeper than the slope of the same distribution for the IP component.

\section{CONCLUSIONS}

The results obtained so far by us suggest that the physical conditions in the regions responsible for HFCs emission might be similar to those in the main pulse (MP) and 
interpulse (IP) emission regions. This idea is supported not only by our detection of GRPs phenomenon at HFCs phases, but also by their polarization characteristics. Still, the origin of high frequency components is an open question. One of the proposition can be an inward emission [14] that for the Crab light curve produce two, properly placed and of good separation, additional peaks.

So far, it has been thought that the flux density distribution of giant pulses has powerlaw statistics and that they appear associated with non-thermal high energy emission (PSR B0531+21, PSR B1937+21, PSR B1821-24, and PSR B0540-69). The characteristics of some pulsars, e.g. PSR B1133+16 [15], and the discovery of the Crab's giant radio pulses at phases where no high energy emission is known, might rule out this definition. Other observations however seem to support the relation of high energy and radio emissions. Recently it was found that there is a correlation between X-ray and radio pulses for Vela [16], whereas Shearer et al. [17] have detected a correlation between optical emission and GRPs emission. They found that optical pulses coincident with radio giant pulses were of about $3 \%$ brighter on average.

\section{ACKNOWLEDGMENTS}

Aga Słowikowska was supported by grant KBN 2P03D.004.24. She would like to thank Bronek Rudak for his very bright suggestions.

\section{REFERENCES}

1. D. H. Staelin, and E. C. Reifenstein, Science 162, 1481-1483 (1968).

2. J. F. R. Gower, and E. Argyle, ApJ 171, L23-L26 (1972).

3. S. C. Lundgren, J. M. Cordes, M. Ulmer, S. M. Matz, S. Lomatch, R. S. Foster, and T. Hankins, ApJ 453, 433-445 (1995).

4. S. Sallmen, D. C. Backer, T. H. Hankins, D. Moffett, and S. Lundgren, ApJ 517, 460-471 (1999).

5. J. M. Cordes, N. D. R. Bhat, T. H. Hankins, M. A. McLaughlin, and J. Kern, ApJ 612, 375-388 (2004).

6. A. Jessner, A. Słowikowska, B. Klein, H. Lesch, C. H. Jaroschek, G. Kanbach, and T. H. Hankins, Advances in Space Research 35, 1166-1171 (2005).

7. D. A. Moffett, and T. H. Hankins, ApJ 468, 779-783 (1996).

8. D. A. Moffett, and T. H. Hankins, ApJ 522, 1046-1052 (1999).

9. A. Karastergiou, A. Jessner, and R. Wielebinski, "High-frequency Polarimetric Observations of the Crab Pulsar," in IAU Symposium, 2004, pp. 329-330.

10. J. M. Rankin, D. B. Campbell, R. B. Isaacman, and R. R. Payne, A\&A 202, 166-172 (1988).

11. J. M. Weisberg, J. M. Cordes, B. Kuan, K. E. Devine, J. T. Green, and D. C. Backer, ApJS 150, 317-341 (2004).

12. R. T. Edwards, and B. W. Stappers, $A \& A$ 421, 681-691 (2004).

13. E. Argyle, and J. F. R. Gower, ApJ 175, L89-L91 (1972).

14. K. S. Cheng, M. Ruderman, and L. Zhang, ApJ 537, 964-976 (2000).

15. M. Kramer, A. Karastergiou, Y. Gupta, S. Johnston, N. D. R. Bhat, and A. G. Lyne, $A \& A$ 407, 655-668 (2003).

16. J. Donovan, A. Lommen, Z. Arzoumanian, A. Harding, M. Strickman, C. Gwinn, R. Dodson, P. McCulloch, and D. Moffett, "Correlations Between X-ray and Radio Pulses in Vela," in IAU Symposium, 2004, pp. 335-336. 
17. A. Shearer, B. Stappers, P. O'Connor, A. Golden, R. Strom, M. Redfern, and O. Ryan, Science 301, 493-495 (2003). 